\title{
SENSOR GRAPHS FOR GUARANTEED COOPERATIVE LOCALIZATION PERFORMANCE
}

\author{
Y. Yuan* and H. G. Tanner** \\ ${ }^{*}$ Control Group, Department of Engineering, University of Cambridge, \\ Cambridge CB2 1PZ, UK \\ ** Department of Mechanical Engineering, University of Delaware, \\ Newark, DE 19711, USA
}

\begin{abstract}
A group of mobile robots can localize cooperatively, using relative position and absolute orientation measurements, fused through an extended Kalman filter (EKF). The topology of the graph of relative measurements is known to affect the steady state value of the position error covariance matrix. Classes of sensor graphs are identified, for which tight bounds for the trace of the covariance matrix can be obtained based on the algebraic properties of the underlying relative measurement graph. The string and the star graph topologies are considered, and the explicit form of the eigenvalues of error covariance matrix is given. More general sensor graph topologies are considered as combinations of the string and star topologies, when additional edges are added. It is demonstrated how the addition of edges increases the trace of the steady state value of the position error covariance matrix, and the theoretical predictions are verified through simulation analysis.
\end{abstract}

Key words: Cooperative localization, formations, Kalman filter 


\section{Introduction}

In multi-robot systems, robots are often required to work cooperatively to perform a certain task [1-3], and then robot localization is important. Information fusion by means of distributed Kalman filters has been examined both in the case of static [4] and mobile [5] sensor and actuator networks. In cases where robots build sensor data maps [6], for example, measurements are of little use if they cannot be associated with physical locations. If the robots do not have access to absolute position and orientation measurements, for instance in GPS-denied or unstructured indoor environments, they have to rely on relative measurements to localize themselves. Such cases arise in robotic urban surveillance and mapping, emergency response, underground or even extraterrestrial planetary exploration.

Take for example the scenario described in [7] where robots are called to investigate the possibility that illicit radioactive material has been processed, by searching for small specks of fissile material. They are deployed in an indoor area which is not structured to provide them with absolute position information (by setting up a motion capture system, for example). Then, robots are to rely on relative measurements to localize, possibly fused through an EKF. To minimize position estimate errors, it might be beneficial for them to move while holding a specific formation. A single base station (could be one of the robots) may be stationed at a location (e.g. window/door) and serve as a landmark for the other robots. The rest of the robots would move in a formation pattern that gives rise to a particular network of relative measurements, in other words, a special sensing graph.

The contribution of this paper is to expose some of these sensing network topologies based on the system model in Section 2, and analytically show how the topology of the network can affect the upper bound of the steady state value for the trace of the covariance matrix, as established in [8]. To ensure that network topology modifications have an impact on performance, a conservative range for the values of the trace bound is evaluated: the upper limit is indicative of the performance improvement that can be achieved through the particular way of networking; an analysis of the lower limit in Section 3 ensures that the 
trace is not forced to increase as its upper limit decreases, leaving the former some "room" at the bottom. In Section 4, two special sensing graph topologies the string (robots sensing each other in series) and the star (all robots measuring their location with respect to a single other robot) are considered here analytically to derive the explicit form of all the eigenvalues in the error covariance matrix. Finally, more general graphs are considered in Sections 5 and 6 by combining graphs of the two topologies analyzed and by adding new edges.

\subsection{Literature overview}

It has been demonstrated [5], that when the robots in the group use a combination of absolute and relative position measurements, localization accuracy increases. The general perception is that more measurements are better; however, when the cost of measurement, communication, and/or computation is considered, this may not be the case. One option is to develop a reduced order model Kalman filter, without resorting to model reduction [9]. If this is not possible, other trade-offs between performance and resource utilization are sought. In the context of cooperative localization, the question that arises is how can one obtain a sufficiently good level of accuracy with the smallest number of relative measurements [10]. Our motivation comes from the formulation in [8], where the steady state values for the covariance matrix of the position errors in a cooperative EKF implementation are obtained. In [11] it is shown that the trace of this covariance matrix is a non-convex function of the sensing graph topology, and the trace is minimized by using a genetic algorithm. The work in [10] attempts to link more explicitly the covariance matrix trace with the sensor graph topology, by identifying a weighted Laplacian of the sensing graph in the expression of the steady state covariance matrix presented in [8].

Robot localization is frequently linked with (topological) map building in simultaneous localization and mapping (SLAM) [12-14]. In multi-robot teams, cooperative localization

and mapping relies on (extended) Kalman filters [5,8], particle filters [15, 16], grid-based methods [17,18] and expectation maximization algorithms [19,20]. In [21], a continuoustime, closed-form solution to both the single and multiple platform SLAM is given. The 
problem addressed in this paper, however, is not C-SLAM, for we assume the environment (in this particular case, a single landmark) to be known accurately. Rather, this paper focuses on improving robot localization by means of (selective) relative position measurements between robots. Related problems have also been investigated by the sensor network and data fusion communities (see [22-24] among others), although the problems treated in these references are more of a static nature, with the sensor nodes being immobile.

\subsection{Scope and Contribution}

Relating localization performance measures to the topology of the relative measurement (sensor) graph analytically appears to be prohibitively complex for the general case. Maximizing such performance measures with respect to measurement topology can be formalized and treated as a combinatorial optimization problem. This paper adopts a different approach, by not attacking the problem directly. Instead, the research objective here is to analytically derive (under)approximations of the performance achieved when certain sensor graph topologies are implemented.

Specifically, starting from the extended Kalman filter propagation equations as developed in [8], and using the steady state covariance matrix solution derived for that system, the steady state solution is algebraically manipulated to arrive at a form where several inequalities of the Cauchy-Schwarz type can be applied. Their application yields upper and lower bounds for the trace of the covariance matrix. The bounds assume some homogeneity among robots, and exploit the structure of the matrices involved using matrix analysis results [25-27]. They link directly the trace of a reduced order, scaled weighted sensor graph Laplacian, exploiting the structure. It is shown that when some specific, but nontrivial, sensor graph topologies are used, the results can be sharpened to the point where aspects of the sensor graph topology can be directly linked to the localization performance measures. 


\section{An EKF Model for Unicycle-type Robots}

The analysis in this paper builds on the results of [8], in which an extended Kalman filter is developed for estimating the position of a group of robots. This section introduces notation, and states the previously established results on the characterization of cooperative localization accuracy in terms of the trace of the combined system's error covariance matrix. For the details of the derivations that follow, we refer to [8].

\subsection{Position propagation}

Consider $M$ mobile robots, deployed in a two dimensional planar workspace. The robots obtain relative position information from a single landmark, the position of which is assumed to be known accurately, and are assumed to be able to measure their (absolute) orientation directly. The position of robot $i$ is denoted $X_{i}=\left[x_{i}, y_{i}\right]^{T}$, and its orientation $\phi_{i}$. If one adopts a unicycle-type kinematic model for mobile robot $i$, then in discrete-time, the kinematics of robot $i$ are approximated as follows:

$$
\begin{aligned}
& x_{i}(k+1)=x_{i}(k)+V_{i}(k) \delta \cos \left(\phi_{i}(k)\right) \\
& y_{i}(k+1)=y_{i}(k)+V_{i}(k) \delta \sin \left(\phi_{i}(k)\right)
\end{aligned}
$$

where $\delta$ is the time period, $V_{i}$ is the linear speed of robot $i$ and $\omega_{i}$ is angular speed. Position estimates are denoted $\hat{X}$, and position estimation errors $\tilde{X}$. Equations (1) are linearized along the robot's trajectory, and Gaussian, zero mean measurement noise $W_{i}(k)=\left[w_{V_{i}}(k), \tilde{\phi}_{i}(k)\right]^{T}$ is included for speed and orientation measurements, with variance $\sigma_{V_{i}}^{2}$ and $\sigma_{\phi_{i}}^{2}$, respectively. In [28], it is been shown that the covariance matrix of the system noise affecting the $i$ th robot is $Q_{r_{i}}(k)=\mathbb{E}\left\{G_{r_{i}}(k) W_{i}(k) W_{i}^{T} G_{r_{i}}(k)\right\}$ where $\mathbb{E}$ denotes expectation, and $G_{r_{i}}$ is the error propagation matrix associated with the linearized dynamics $\tilde{X}_{i_{k+1 \mid k}}=$ $I_{2 \times 2} \tilde{X}_{i_{k \mid k}}+G_{i}(k) W_{i}(k)$ of robot $i[8]$. After some mathematical derivations (see [28]), and

setting $^{1} G=\left[\begin{array}{ll}I_{2 M \times 2 M} & 0_{2 N \times 2 M}\end{array}\right]^{\top}$ the covariance propagation equations of the state error is

\footnotetext{
${ }^{1}$ Matrix $G$ should not be confused with $G_{r_{i}}$.
} 
written $P_{k+1 \mid k}=P_{k \mid k}+Q(k)=P_{k \mid k}+G Q_{r}(k) G^{T}$, where $P_{k+1 \mid k}$ and $P_{k \mid k}$ are the covariance of the position errors in estimates $X(k+1)$ and $X(k)$ respectively, conditioned on sensor information available at time step $k$.

\subsection{Position update}

In addition to speed and orientation measurements, the EKF of the previous section uses relative position measurements between robots, and between robots and landmarks. The EKF covariance update equation takes the form $P_{k+1 \mid k+1}=P_{k+1 \mid k}-P_{k+1 \mid k} H^{T} S^{-1} H P_{k+1 \mid k}$ [8]. As it turns out [10], $H$ is actually obtained from the incidence matrix $B$ of the oriented relative sensing graph:

Definition 1. The relative sensing graph is an oriented graph $\mathcal{G}=\{\mathcal{V}, \mathcal{E}\}$, where

- $\mathcal{V}=\{0,1, \ldots, M\}$ is the set of vertices, indexing $M$ robots and one landmark, and

- $\mathcal{E} \subseteq \mathcal{V} \times \mathcal{V}$ is a set of ordered pairs, denoting oriented edges in the graph and expressing relative measurements. The head of each edge is the index of the object being observed, and the tail corresponds to the robot making the observation.

\subsection{Covariance matrix for accurate landmark location}

The system of the $M$ robots with kinematics expressed by (1) moving in a workspace with one landmark, becomes observable if the absolute position of the landmark is available. The same analysis applies to cases where one robot has access to its absolute position information. The steady-state solution to Riccati recursion is shown in [8] to be:

$$
P_{\infty}^{u(0)}=\left[\begin{array}{cc}
P_{r r_{\infty}}^{u} & 0_{2 M \times 2} \\
0_{2 \times 2 M} & 0_{2 \times 2}
\end{array}\right]
$$


The trace of $P_{\infty}^{u(0)}$ serves as a measure of localization accuracy. Note that $\operatorname{trace}\left\{P_{\infty}^{u(0)}\right\}=$ trace $\left\{P_{r r_{\infty}}^{u}\right\}$, and matrix $P_{r r_{\infty}}^{u}$ being symmetric it can be decomposed as:

$$
P_{r r_{\infty}}^{u}=Q_{r_{u}}^{\frac{1}{2}} U \operatorname{diag}\left\{\frac{1}{2}+\left(\frac{1}{4}+\frac{1}{\lambda_{i}}\right)^{\frac{1}{2}}\right\} U^{T} Q_{r_{u}}^{\frac{1}{2}}
$$

where $Q_{r_{u}}=\operatorname{diag}\left\{q_{i}\right\}, q_{i} \triangleq \max \left\{\delta^{2} \sigma_{V_{i}}^{2}, \delta^{2} V_{i}^{2} \sigma_{\phi_{i}}^{2}\right\}$, matrix $U$ is formed by the eigenvectors of:

$$
\psi \triangleq Q_{r_{u}}^{\frac{1}{2}} I_{r} Q_{r_{u}}^{\frac{1}{2}}
$$

and $\lambda_{i}$ are the eigenvalues of $\psi$. Matrix $I_{r}$ that appears in (4) is defined as:

$$
I_{r}=\left[\begin{array}{ll}
I_{2 M \times 2 M} & 0_{2 M \times 2}
\end{array}\right] H^{T} R_{u}^{-1} H\left[I_{2 M \times 2 M} 0_{2 M \times 2}\right]^{T}
$$

where $R_{u} \triangleq \operatorname{diag}\left\{\left(\sigma_{\rho_{i}}^{2}+M_{i} \sigma_{\phi_{i}}^{2} \rho_{0}^{2}+\sigma_{\theta_{i}}^{2} \rho_{0}^{2}\right) I_{2 M_{i} \times 2 M_{i}}\right\}$, with $\rho_{0}$ being the maximum distance at which relative measurements can be recorded by any robot.

\section{General Bounds for the Covariance Trace}

This section presents general bounds for the trace of the covariance matrix (2) which can be tight under certain conditions. In (3), assume for simplicity that $Q_{r_{u}}=q I$, that is, $q_{i}=q$ $\forall i \in\{1, \ldots, M\}$. This would correspond to the case where all robots have identical sensors and are moving with the same translational speed. From (2) and (3) it follows:

$$
\operatorname{trace}\left(P_{r r_{\infty}}^{u(0)}\right)=\operatorname{trace}\left(U^{T} Q_{r_{u}}^{\frac{1}{2}} Q_{r_{u}}^{\frac{1}{2}} U \operatorname{diag}\left\{\frac{1}{2}+\left(\frac{1}{4}+\frac{1}{\lambda_{i}}\right)^{\frac{1}{2}}\right\}\right)=q \sum_{i=1}^{M}\left[\frac{1}{2}+\left(\frac{1}{4}+\frac{1}{\lambda_{i}}\right)^{\frac{1}{2}}\right]
$$

Having assumed that $q=q_{i}$ is a constant, the only way to affect the value of the trace is to manipulate $\sum_{i} q\left(\frac{1}{4}+\frac{1}{\lambda_{i}}\right)^{\frac{1}{2}}$. Matrix $\psi$ is positive definite as a maximal principal submatrix of a weighted Laplacian matrix of the sensor graph. The following theorem establishes upper and lower bounds for the steady state value of the cooperative extended Kalman filter's covariance matrix. The derived bounds depend on the trace and spectral condition number of the maximal principal submatrix $\psi$ of the sensor graph's weighted Laplacian, and the size of the robot group $M$. 
Theorem 1. If $M$ robots have identical omnidirectional sensors, update their state at time intervals equal to $\delta$, and move with the same translational speed $V$ in an environment with one accurately known landmark, then the trace of the steady state covariance matrix of (2) is upper and lower bounded by a function of the trace of the reduced weighted Laplacian matrix of the sensor graph as follows:

$$
\frac{M q}{2}+M q\left(\frac{1}{4}+\frac{M}{\operatorname{trace}(\psi)}\right)^{\frac{1}{2}} \leq \operatorname{trace}\left(P_{\infty}^{u(0)}\right) \leq \frac{M q}{2}+M q\left(\frac{1}{4}+\frac{(\kappa+3)}{4} \frac{M}{\operatorname{trace}(\psi)}\right)^{\frac{1}{2}}
$$

where $\psi$ is a maximal principal submatrix of the sensor graph's weighed Laplacian matrix defined in (4), $q \triangleq \max \left\{\delta^{2} \sigma_{V}^{2}, \delta^{2} V^{2} \sigma_{\phi}^{2}\right\}, \sigma_{V}^{2}$ and $\sigma_{\phi}^{2}$ are the variances on the robots' speed and orientation measurements, respectively, and $\kappa$ is the spectral condition number of $\psi$.

Proof. To derive the lower bound, define the real function $f(x)=\left(\frac{1}{4}+\frac{1}{x}\right)^{\frac{1}{2}}$, for $x>0$, and note that its second derivative is positive definite. This means that $f(x)$ is a convex function, and using the Jensen inequality $\left(\frac{1}{4}+\frac{1}{\sum_{i=1}^{M} \frac{\lambda_{i}}{M}}\right)^{\frac{1}{2}} \leq \sum_{i=1}^{M}\left(\frac{1}{4}+\frac{1}{\lambda_{i}}\right)^{\frac{1}{2}} \frac{1}{M}$, it follows that $M\left(\frac{1}{4}+\frac{M}{\operatorname{trace}(\psi)}\right)^{\frac{1}{2}} \leq \sum_{i=1}^{M}\left(\frac{1}{4}+\frac{1}{\lambda_{i}}\right)^{\frac{1}{2}}$. Substituting into (4.1), yields the lower bound of (7). To derive the upper bound, apply the Cauchy-Schwarz inequality on vectors $(q, \ldots, q)^{T}$ and $\left(\frac{1}{4}+\frac{1}{\lambda_{1}}, \ldots, \frac{1}{4}+\frac{1}{\lambda_{M}}\right)^{T}$ to get $\sum_{i=1}^{M} q\left(\frac{1}{4}+\frac{1}{\lambda_{i}}\right)^{\frac{1}{2}} \leq q\left[M\left(\frac{M}{4}+\sum_{i=1}^{M} \frac{1}{\lambda_{i}}\right)\right]^{\frac{1}{2}}$. Now using Kantorovich's inequality, one obtains $\left(\sum_{i=1}^{M} \frac{1}{\lambda_{i}}\right)\left(\sum_{i=1}^{M} \lambda_{i}\right) \leq \frac{M^{2}}{4}\left(\frac{\lambda_{\max }}{\lambda_{\min }}+2+\frac{\lambda_{\min }}{\lambda_{\max }}\right)$, where one can identify $\frac{\lambda_{\max }}{\lambda_{\min }}$ as the spectral condition number $\kappa$, and $\sum_{i=1}^{M} \lambda_{i}$ as $\operatorname{trace}(\psi)$, so that the inequality can be written as $\sum_{i=1}^{M} \frac{1}{\lambda_{i}} \leq \frac{M^{2}\left(\kappa+\kappa^{-1}+2\right)}{4 \operatorname{trace}(\psi)} \leq \frac{M^{2}(\kappa+3)}{4 \operatorname{trace}(\psi)}$, given that $\kappa \leq 1$. Substituting the right hand side in to the right hand side of the Cauchy-Schwarz inequality, and using (4.1), the right hand side of (7) is obtained.

The upper and lower bounds in (7) are quite similar in form, and have the trace of $\psi$ appearing only in the denominator. The significance of the latter is that as the eigenvalues of this principal sub-matrix of the weighted Laplacian are increased, both the upper and lower bound decrease. For if there is a significant gap between the two bounds, there is always the possibility that $\operatorname{trace}\left(P_{\infty}^{u(0)}\right)$ increases as trace $(\psi)$ increases, while still satisfying (7). In principle, however, (7) eventually forces $\operatorname{trace}\left(P_{\infty}^{u(0)}\right)$ to decrease for a sufficiently large $\operatorname{trace}(\psi)$. 


\section{Special Topologies}

In this section two special topologies, which appear frequently in practical formation control applications, are considered.

\subsection{The String}

One of the simplest interconnection topologies is that of a string (single path): one robot observes the known landmark, and all other robots observe a single other robot. Let the string be a path of length $n$, and consider the single-dimensional case for simplicity. (Results can be extended to the multi-dimensional case, using the properties of the Kronecker product.) Define $w_{k} \triangleq\left(\sigma_{\rho}^{2}+k \sigma_{\phi}^{2} \rho_{0}^{2}+\sigma_{\theta}^{2} \rho_{0}^{2}\right)^{-1}$, and rewrite (5) in the form:

$$
I_{r}=\left[\begin{array}{cccccc}
3 w_{2} & -2 w_{2} & 0 & 0 & \cdots & 0 \\
-2 w_{2} & 4 w_{2} & -2 w_{2} & 0 & \cdots & 0 \\
\vdots & \vdots & \ddots & \ddots & & -w_{1}-w_{2} \\
0 & 0 & 0 & \cdots & -w_{1}-w_{2} & w_{1}+w_{2}
\end{array}\right]
$$

Let us define matrix $N$ such that $I_{r}=2 w_{2} N$ where:

$$
N \triangleq\left[\begin{array}{ccccc}
\frac{3}{2} & -1 & 0 & \cdots & 0 \\
-1 & 2 & -1 & & \\
\vdots & & \ddots & \ddots & \\
0 & & & -a & a
\end{array}\right]
$$

where $a=\frac{w_{1}+w_{2}}{2 w_{2}}>1$. If $\lambda$ is an eigenvalue of $N$, then $2 w_{2} \lambda$ is an eigenvalue of $I_{r}$. The Geršgorin disc theorem ensures that all eigenvalues of $N$ satisfy $\lambda \leq 4$.

Lemma 1. The eigenvalues $\lambda$ of the $n$-dimensional matrix $N$ defined in (9), for any $n>3$, are the solutions of the algebraic equation

$$
\tan ((n-3) \zeta(\lambda))=\left(\frac{\sqrt{4 \lambda-\lambda^{2}}\left(\lambda^{2}-(2 a+1) \lambda+a\right)}{\lambda\left(-\lambda^{2}+(2 a+3) \lambda-5 a\right)}+\frac{\lambda-\frac{1}{2}}{\sqrt{4 \lambda-\lambda^{2}}}\right)^{-1}
$$

where $\tan \zeta(\lambda)=\frac{\sqrt{4 \lambda-\lambda^{2}}}{2-\lambda}$.

Proving Lemma 1 is facilitated by the following lemma: 
Lemma 2. Consider a sequence $\left\{X_{n}\right\}_{n=0}^{\infty}$, with $X_{i} \in \mathbb{R} \forall i$, defined by the recursive equation:

$$
X_{k}=\Sigma X_{k-1}+\Omega X_{k-2}, \quad k \geq 2
$$

for which $\Sigma, \Omega \in \mathbb{R}$ and $X_{0}, X_{1}$ are given. Then the elements in the sequence can be expressed as $X_{k}=\mu \delta_{1}^{k}+\nu \delta_{2}^{k}$, where $\delta_{1}, \delta_{2} \in \mathbb{C}$ are the roots of the polynomial $x^{2}-\Sigma x-\Omega=0$, and $\mu$ and $\nu$ are complex numbers determined by the two initial conditions.

Proof of Lemma 2. The statement is proved by induction. Proving the base case is straightforward. Assume that the general case holds for $X_{1}, X_{2}, \cdots, X_{k}, k \geq 2$, and let $X_{k+1}$ be given by equation (10). It follows: $X_{k+1}=\Sigma X_{k}+\Omega X_{k-1}=\mu \delta_{1}^{k-1}\left(\Omega+\Sigma \delta_{1}\right)+\nu \delta_{2}^{k-1}\left(\Omega+\Sigma \delta_{2}\right)=$ $\mu \delta_{1}^{k+1}+\nu \delta_{2}^{k+1}$, where the last equation holds because $\forall i, \Sigma \delta_{i}+\Omega=\delta_{i}^{2}$ by definition. With the induction argument made, the proof is completed.

Proof of Lemma 1. Since the eigenvalues of $N$ are roots of $\operatorname{det}(N-\lambda I)$, and given that $N$ is positive definite, $\lambda \in \mathbb{R}$. Define a sequence of matrices $\left\{\Psi_{i}\right\}_{0}^{n}$ of increasing dimension $i$ :

$$
\Psi_{k} \triangleq\left[\begin{array}{ccccc}
\frac{3}{2}-\lambda & -1 & 0 & \cdots & 0 \\
-1 & 2-\lambda & -1 & & \\
0 & -1 & 2-\lambda & -1 & \\
\vdots & & & \ddots & \\
0 & & & -1 & 2-\lambda
\end{array}\right] \in \mathbb{R}^{k \times k}
$$

Since $\operatorname{det}\left(\Psi_{k}\right)=(2-\lambda) \operatorname{det}\left(\Psi_{k-1}\right)-\operatorname{det}\left(\Psi_{k-2}\right)$, one has:

$$
\operatorname{det}(N-\lambda I)=-a^{2} \operatorname{det}\left(\Psi_{n-2}\right)+(a-\lambda) \cdot\left[(1+a-\lambda) \operatorname{det}\left(\Psi_{n-2}\right)-\operatorname{det}\left(\Psi_{n-3}\right)\right]
$$

The first three matrices in sequence $\left\{\Psi_{i}\right\}_{0}^{n}$ are:

$$
\operatorname{det}\left(\Psi_{0}\right)=1 \quad \operatorname{det}\left(\Psi_{1}\right)=\frac{3}{2}-\lambda \quad \operatorname{det}\left(\Psi_{2}\right)=\lambda^{2}-\frac{7}{2} \lambda+2
$$

and Lemma 2 implies that:

$$
\operatorname{det}\left(\Psi_{k}\right)=(f+b j)(c+d j)^{k}+(f-b j)(c-d j)^{k}
$$


where $j \triangleq \sqrt{-1}$, and $c+d j, c-d j$ are the roots of $x^{2}-(2-\lambda) x+1=0$, in which $c=\frac{2-\lambda}{2}$ and $d=\frac{\sqrt{4 \lambda-\lambda^{2}}}{2}$. Constants $f$ and $b$ can be derived from $(12): f=\frac{1}{2}$, and $b=\frac{\lambda-\frac{1}{2}}{2 \sqrt{4 \lambda-\lambda^{2}}}$. Defining $\beta$ and $\zeta$ as $\tan \beta \triangleq \frac{b}{f}$ and $\tan \zeta \triangleq \frac{d}{c}$, allows one to write $c \pm d j=\cos \zeta+j \sin \zeta$, because $c^{2}+d^{2}=1$. Rename the conjugate pairs in (13) as $A \triangleq f+b j, \bar{A}=f-b j, B \triangleq c+d j$, $\bar{B}=c-d j$, and set $r=\sqrt{f^{2}+b^{2}}>0$. Then, by manipulating right hand side of (11):

$$
\begin{aligned}
\operatorname{det}(N-\lambda I)=A & B^{n-3} \cdot\left(\frac{-\lambda^{3}+(2 a+3) \lambda^{2}-5 a \lambda}{2}+j \frac{\sqrt{4 \lambda-\lambda^{2}}\left[\lambda^{2}-(2 a+1) \lambda+a\right]}{2}\right) \\
& +\bar{A} \bar{B}^{n-3} \cdot\left(\frac{-\lambda^{3}+(2 a+3) \lambda^{2}-5 a \lambda}{2}-j \frac{\sqrt{4 \lambda-\lambda^{2}}\left[\lambda^{2}-(2 a+1) \lambda+a\right]}{2}\right)
\end{aligned}
$$

and setting: $R \triangleq \frac{\sqrt{\left(-\lambda^{3}+(2 a+3) \lambda^{2}-5 a \lambda\right)^{2}+\left(4 \lambda-\lambda^{2}\right)\left(\lambda^{2}-(2 a+1) \lambda+a\right)^{2}}}{2} \neq 0, \tan \alpha \triangleq \frac{\left(\lambda^{2}-(2 a+1) \lambda+a\right) \sqrt{4 \lambda-\lambda^{2}}}{-\lambda^{3}+(2 a+3) \lambda^{2}-5 a \lambda}$, one rewrites the characteristic equation of (14) as:

$$
2 R r \cos (\alpha+\beta+(n-3) \zeta)=0
$$

Since the eigenvalues $\lambda$ of $N$ satisfy (15), and given that neither $R$ nor $r$ are zero:

$$
\cos (\alpha+\beta+(n-3) \zeta)=0 \Rightarrow \tan ((n-3) \zeta(\lambda))=\frac{1}{\tan (\alpha(\lambda)+\beta(\lambda))}
$$

Substitution completes the proof.

Using Lemma 1 one can prove the following Proposition for the case of the string.

Proposition 1. Under the assumptions of Theorem 1, if the relative sensing graph is a path, then the smallest eigenvalue of $I_{r}$ decreases with the length of the path.

Proof. Lemma 1 suggests that for a string of length $n>3, \tan ((n-3) \zeta(\lambda))=\frac{1}{\tan (\alpha(\lambda)+\beta(\lambda))}$. Suppose a new node is connected at the end of the string. Then:

$$
\tan \left((n-2) \zeta\left(\lambda^{\prime}\right)\right)=\frac{1}{\tan \left(\alpha\left(\lambda^{\prime}\right)+\beta\left(\lambda^{\prime}\right)\right)}
$$

where $\lambda^{\prime}$ is an eigenvalue of the $I_{r}$ matrix for the augmented string. The strategy here is to compare the smallest eigenvalue of the two graphs using (16). A rough approximation could easily be made that this smallest eigenvalue satisfies $\lambda \equiv \lambda_{\min }<1 / 2$, given $a>1$ and $n=4$. 
Manipulating (16) yields $\tan \left((n-2) \zeta\left(\lambda^{\prime}\right)\right)=\frac{\tan \left((n-3) \zeta\left(\lambda^{\prime}\right)\right)+\tan \left(\zeta\left(\lambda^{\prime}\right)\right)}{1-\tan \left(\zeta\left(\lambda^{\prime}\right)\right) \tan \left((n-3) \zeta\left(\lambda^{\prime}\right)\right)}$. Define $\varphi(x) \triangleq$ $\tan ((n-3) \zeta(x))-\frac{1}{\tan (\alpha(x)+\beta(x))}$, which is increasing with $x$. To see this, differentiate the first term in the expression of $\phi(x)$ :

$$
\frac{\mathrm{d} \tan ((n-3) \zeta(x))}{\mathrm{d} x}=\frac{\frac{(n-3)\left(2+7 x-2 x^{2}\right)}{2(2-x)^{2} \sqrt{4 x-x^{2}}}}{\left(1+\tan ^{2}(\zeta(x))\right) \cos ^{2}((n-3) \zeta(x))} \geq 0, \quad \text { for } x<1
$$

For the second term, one has:

$$
\begin{aligned}
& \frac{\mathrm{d}}{\mathrm{d} x}(\tan (\alpha(x))+\tan (\beta(x)))=\frac{x\left(x^{2}-(2 a+1) x+a\right)^{2}}{4 x-x^{2}+\left(-x^{3}+(2 a+3) x^{2}-5 a x\right)^{2} \sqrt{4 x-x^{2}}} \\
& \cdot\left[-\left(8 a^{2}+8 a\right) x^{3}+\left(32 a^{2}-34 a+6\right) x^{2}+\left(8 a^{2}-8 a\right) x+10 a^{2}\right]+\frac{1+\frac{3}{2} x}{\sqrt{4 x-x^{2}}\left[3 x+\frac{1}{4}\right]}
\end{aligned}
$$

Recall that $a>1$ as in the definition, both two terms in the right hand side of the above expression are non-negative for all $0<x<1 / 2$. Since both terms in the expression of $\varphi(x)$ are non decreasing, the function is non decreasing too in $(0,1 / 2)$. Note that by Lemma 1 , $x=\lambda \Rightarrow \varphi(x)=0$. For $x=\lambda^{\prime}, \varphi\left(\lambda^{\prime}\right)=-\frac{\tan \left(\zeta\left(\lambda^{\prime}\right)\right)\left[\tan ^{2}\left((n-3) \zeta\left(\lambda^{\prime}\right)\right)+1\right]}{1-\tan \left(\zeta\left(\lambda^{\prime}\right)\right) \tan \left((n-3) \zeta\left(\lambda^{\prime}\right)\right)}$, Since $\tan (\zeta(x))=$ $\frac{\sqrt{4 x-x^{2}}}{2-x}$, when $x \rightarrow 0^{+}$it follows that $\zeta(x) \rightarrow 0^{+}$, thus the numerator of $\varphi\left(\lambda^{\prime}\right)$ is positive. A direct calculation of $\tan \left(\zeta\left(\lambda^{\prime}\right)\right) \tan \left((n-3) \zeta\left(\lambda^{\prime}\right)\right)$ verifies that for $a>1$ and $\lambda^{\prime} \rightarrow 0^{+}$, the expression is negative in a neighborhood of zero (the width of which depends on $a$ ), and maintains a negative derivative. Thus, as length of the string is increasing and $\lambda^{\prime} \rightarrow 0^{+}$, given that the denominator of $\varphi\left(\lambda^{\prime}\right)$ is positive, one has $\varphi\left(\lambda^{\prime}\right)<0$. Therefore, with $\varphi(x)$ being an increasing function near zero, and with $\varphi\left(\lambda^{\prime}\right) \leq \varphi(\lambda) \equiv 0$ it follows that $\lambda^{\prime} \leq \lambda$. In other words, as the length of the string increases, the smallest eigenvalue of $I_{r}$ decreases.

The significance of Proposition 1 is since in (4.1), $\operatorname{trace}\left(P_{r r_{\infty}}^{u(0)}\right)=q \sum_{i=1}^{M}\left[\frac{1}{2}+\left(\frac{1}{4}+\frac{1}{\lambda_{i}}\right)^{\frac{1}{2}}\right]$, the inverse of the smallest eigenvalue of $\psi, \min _{i}\left\{\lambda_{i}\right\}$, (which is directly proportional to the corresponding eigenvalue of $I_{r}$ ) is the dominant part of the expression. As expected, when the string gets longer the localization performance suffers. 


\subsection{The Star}

The star configuration considered in this paper is one where all robots are associated with leaves of a sensor graph which is tree of depth two, except for one which directly observes the landmark. For this case, matrix $I_{r}$ can take the form:

$$
I_{r}=\left[\begin{array}{cccc}
n w_{n}+(n-1) w_{1} & -w_{1}-w_{n} & \ldots & -w_{1}-w_{n} \\
-w_{1}-w_{n} & w_{1}+w_{n} & \ldots & 0 \\
\vdots & & \ddots & \vdots \\
-w_{1}-w_{n} & 0 & \ldots & w_{1}+w_{n}
\end{array}\right]
$$

and one can show the following:

Lemma 3. As $n$ increases, the smallest eigenvalue of $I_{r}$ approaches $\lambda=\frac{n w_{1}+(n+1) w_{n}-\sqrt{\Delta}}{2}$, where $\Delta=\left(n^{2}+2 n-3\right) w_{n}^{2}+n^{2} w_{1}^{2}+2\left(n^{2}+n-2\right) w_{1} w_{n}$, for which one has $\lim _{n \rightarrow \infty} \Delta(n)=0$.

Proof. For a Hermitian matrix of the form $A=\left(\begin{array}{ll}a & x^{*} \\ x & \bar{A}\end{array}\right) \in \mathbb{C}^{n \times n}$ in which $a \in \mathbb{C}, x \in$ $\mathbb{C}^{n-1}$ and $\bar{A} \in M_{n-1}$, one has $\operatorname{det}(A)=\operatorname{adet}(\bar{A})-x^{*}(\operatorname{adj} \bar{A}) x$. The characteristic polynomial of $I_{r}$ as expressed in (17) has a closed form expression $\operatorname{det}\left(\lambda I-I_{r}\right)=\left(w_{1}+w_{n}-\right.$ $\lambda)^{n-2}\left[\lambda^{2}-\left(n w_{1}+(n+1) w_{n}\right) \lambda+w_{n}^{2}+w_{1} w_{n}\right]$, which implies that the eigenvalues of $I_{r}$ are equal to $\lambda_{1,2}=\frac{n w_{1}+(n+1) w_{n} \pm \sqrt{\Delta}}{2}$, for $\lambda_{3, \ldots, n}=w_{1}+w_{n}$, where $\Delta=\left(n^{2}+2 n-3\right) w_{n}^{2}+n^{2} w_{1}^{2}+$ $2\left(n^{2}+n-2\right) w_{1} w_{n}$. Since $\frac{n w_{1}+(n+1) w_{n}-\sqrt{\Delta}}{2}<\frac{n w_{1}+(n+1) w_{n}+\sqrt{\Delta}}{2}$, it suffices to compare the left hand side to $w_{1}+w_{n}$. As $n \rightarrow \infty$ one has: $\lambda_{2}=\frac{2\left(w_{1}+w_{n}\right) w_{n}}{n w_{1}+(n+1) w_{n}+\sqrt{\Delta}}<\frac{2 w_{n}\left(w_{n}+w_{1}\right)}{n\left(w_{1}+w_{n}\right)}=\frac{2 w_{n}}{n} \rightarrow 0$, as $n \rightarrow \infty$.

Lemma 3 implies that the star configuration yields cooperative localization accuracy that decreases with the size group; however, there is a lower limit which is determined by the smallest eigenvalue of $I_{r}$. This, in turn, converges to 0. Similar to the case of the string, the star interconnection's localization accuracy degrades as the size of the robot team increases. 


\section{The Addition of Graph Edges}

Eigenvalue interlacing theorems [29] generally suggest that the addition of edges in a graph do not decrease the Laplacian eigenvalues. Weighted graphs behave similarly [30], and a proof for this behavior is found in [10]. Sensing graphs, however, are different because changes in the edge set affect the weights on the edges, and therefore the results just mentioned do no

longer hold. Nevertheless, it is shown in [10] that the trace of the (weighted) Laplacian of a sensing graph does not decrease. Along the same lines, it is not too hard to show that the addition of a new vertex adjacent to the new edge, also increases (not monotonically, possibly) the trace:

Corollary 1. Let $X$ be the weighted sensing graph with $n$ vertices, and let $Y$ be obtained from $X$ by adding a new vertex and an edge joining this vertex to a vertex in $X$. Then $\operatorname{trace}(Q(Y)) \geq \operatorname{trace}(Q(X))$.

Although the results for the trace mentioned above are encouraging, due to the changes in the graph weights, it is unclear if one can state similar positive results for the case of the eigenvalues, even for the case where a new vertex is being added. Although the aggregate eigenvalue behavior tends to increase the trace, individual eigenvalues may increase or decrease in general.

\section{The String-Star}

Strings were shown to exhibit cooperative localization accuracy that degrades with the length of the string. On the other hand, star configurations come with localization accuracy that degrades more gracefully, however they cannot practically scale, because there is a limit to how many different other robots can a single robot observe.

This limitation motivates us to consider a "hybrid" topology, which is named string-star. In the string-star topology, we have several strings attached to the single landmark. The 
graph can grow in size along each one of the "hanging" branches. The absence of the single node with depth one, compared to the case of the 2-star, eliminates the possibility of a graceful accuracy degradation; nevertheless, it is anticipated that one can strike a balance between the ability of the topology to scale in practice, and the existence of analytical lower bounds on its localization accuracy.

What makes the the string-star configuration analytically tractable is the fact that the corresponding $I_{r}$ matrix is block diagonal; thus the spectrum is formed by the union of the eigenvalues of its blocks. Each one of these blocks is associated with a different string, and it has the form shown in (8). Following the discussion in Section 4.1 one can obtain a lower bound for the group of eigenvalues in each such block.

Each individual string can grow or shrink, and its associated eigenvalues respect the inequalities of Proposition 1. What is noteworthy, however, that creating a loop across strings may not always increase the eigenvalues. Simulation results indicate that eigenvalues can shift either way. However, the trace of $P_{r r_{\infty}}^{u(0)}$ does increase, which means that additional measurements generally improve accuracy.

Table 1: Changes in $\lambda_{1}$, and trace $\left(P_{r r_{\infty}}^{u(0)}\right)$ for the cases in Fig 1.

\begin{tabular}{||l|c|c|c|c|c|c||}
\hline \hline & $(\mathrm{a})$ & $(\mathrm{b})$ & $(\mathrm{c})$ & $(\mathrm{d})$ & $(\mathrm{e})$ & $(\mathrm{f})$ \\
\hline$\Delta \lambda_{1}$ & 0.83 & -0.05 & -0.01 & 0.32 & -0.27 & 0.65 \\
\hline$\Delta \operatorname{trace}\left(P_{r r_{\infty}}^{u(0)}\right)$ & $0.35 \mathrm{q}$ & $0.23 \mathrm{q}$ & $0.26 \mathrm{q}$ & $0.28 \mathrm{q}$ & $0.15 \mathrm{q}$ & $0.21 \mathrm{q}$ \\
\hline \hline
\end{tabular}

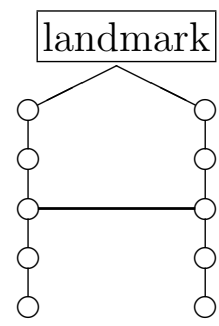

(a)

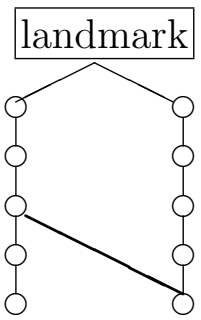

(b)

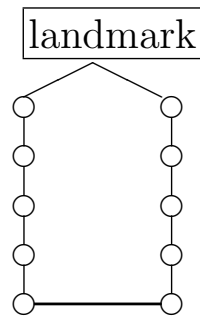

(c)

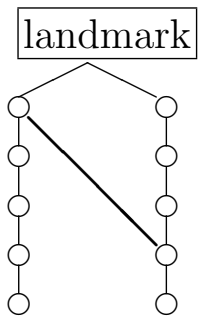

(d)

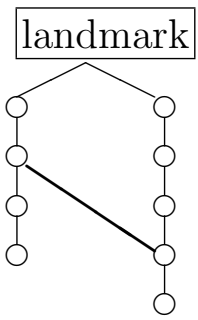

(e)

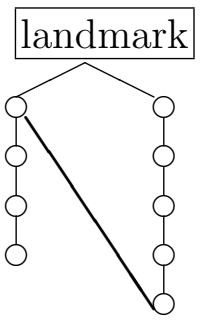

(f)

Figure 1: Several different ways of connecting across strings of the star-string topology. 


\section{Implementation Issues}

The behavior of an experimental testbed on which the approach presented here is implemented, is expected to depend to a great extend on the available hardware. In preliminary studies implementing an EKF under the same assumptions made in this paper, as well as in [8], on the mobile robot used in [7] equipped with an additional compass for absolute orientation information, and using its infrared sensors for distance measurement, problems primarily related to the robot's computational and sensing hardware were encountered [31]. Simulation results reported in [31], however, suggest that a EKF implementation and testing in silico, with simulated Gaussian measurement and system noise variances of 0.0049 and 0.0001 respectively, yield an RMS error between the actual and the estimated position of approximately $500 \mathrm{~mm}$. To avoid instability issues that are inherent to typical EKF implementations, the difference between successive IR distance readings had to be thresholded at $20 \mathrm{~mm}$ (see [32] for details on thresholding).

In [31], only a single robot EKF was implemented, and therefore the conclusions on applicability and performance do not transfer directly to this work. The study, however, indicates the feasibility of the implementation, albeit the availability of accommodating robotic hardware, such as one found in bigger mobile robots available in the market today, capable of hosting a mini-ATX processor.

\section{Summary}

Analytical bounds are derived for the performance of a cooperative localization scheme, based on the extended Kalman filter, for groups of robots that utilize relative position and bearing measurements. For general topologies expressing the network of relative measurements, computing more tight bounds may become extremely complex. Two special sensing graph topologies are studied, and for these particular cases, analytical bounds are derived and the implications are discussed. These two graph topologies are the string (robots sensing each 
other in series) and the star (all robots measuring their location with respect to a single other robot). Analytical results agree with the expectation that performance deteriorates with the length of the string. For the case of the star, however, the analysis suggests a lower bound on the localization performance as the size of the group increases. This effect may deserve additional investigation, as it could potentially offer methods of allowing groups of robots that localize cooperatively to scale in size without affecting the accuracy of their collective estimates.

\section{Acknowledgements}

The work of the second author is supported by the National Science Foundation under Grant \# 00447898.

\section{References}

[1] Lynne E. Parker. Current state of the art in distributed autonomous mobile robotics. In Lynne E. Parker, George Bekey, and Jacob Barhen, editors, Proceedings of the 5th International Symposium on Distributed Autonomous Robotic Systems, pages 3-12. SpringerVerlag, Tokyo, October 2000.

[2] Miguel Schneider-Fontán and Maja J. Matarić. Territorial multirobot task division. IEEE Transactions of Robotics and Automation, 14(5):815-822, 1998.

[3] Y. Arai, T. Fujii, H. Asama, H. Kaetsu, and I. Endo. Realization of autonomous navigation in multirobot environment. In Proceedings of the IEEE/RSJ lnternational Conference on lntelligent Robots and Systems, pages 1999-2004, 1998.

[4] S. L. Sun. Optimal fusion distributed filter for discrete multichannel ARMA signals. Control and Intelligent Systems, 34(1):80-86, 2006. 
[5] S.I. Roumeliotis and G.A. Bekey. Distributed multi-robot localization. Distributed Autonomous Robotic Systems, 4:179-188, 2000.

[6] R. A. Cortez and H. G. Tanner. Radiation mapping using multiple robots. In Proceedings of the 2nd Intl. Joint Topical Meeting on Emergency Preparedness 83 Response and Robotic 83 Remote Systems, 2008.

[7] Herbert G. Tanner R. Andres Cortez and Ron Lumia. Distributed robotic radiation mapping. In G. J. Pappas O. Khatib and V. Kumar, editors, Experimental Robotics - The Eleventh International Symposium, volume 54 of Springer tracts in advanced robotics, pages 147-156. Springer, 2009.

[8] A. Mourikis and S. Roumeliotis. Performance bounds for cooperative simultaneous localization and mapping. In Robotics: Science and Systems, pages 73-80, 2005.

[9] D. Simon. Reduced order Kalman filtering without model reduction. Control and Intelligent Systems, 35(2):169-174, 2007.

[10] D. Kumar and H.G. Tanner. How sensor graph topology affects localization accuracy. In Proceedings of the European Control Conference, pages 868-873, 2007.

[11] Y. Hidaka, A. Mourikis, and S. Roumeliotis. Optimal sensing strategies for mobile robot formations. In Robotics: Science and Systems, pages 281-288, 2005.

[12] M.W.M.G. Dissanayake, P. Newman, S. Clark, H.F. Durrant-Whyte, and M. Csorba. A solution to the simultaneous localization and map building (SLAM) problem. IEEE Transactions on Robotics and Automation, 17(3):229-241, 2001.

[13] H. Choset and K. Nagatani. Topological simultaneous localization and mapping (SLAM): Toward exact localization without explicit localization. IEEE Transactions on Robotics and Automation, 17(2):125-137, 2001.

[14] S. Thrun, W. Burgard, and D. Fox. A probabilistic approach to cuncurrent mapping and localization for mobile robots. Autonomous Robots, 5:1-25, 1998. 
[15] S. Thrun, D. Fox, W. Burgard, and F. Dellaert. Robust monte carlo localization for mobile robots. Artificial Intelligence, 128(1-2):99-141, 2000.

[16] W. Burgard, D. Fox, M. Moors, R. Simmons, and S. Thrun. Collaborative multi-robot exploration. In IEEE International Conference on Robotics and Automation, volume 1, pages 476-481, 2000.

[17] Dieter Fox. Markov Localization: A Probabilistic Framework for Mobile Robot Localization and Navigation. Ph.d dissertation, University of Bonn, 1998.

[18] W. Burgard, D. Fox, D. Henning, and T. Schmidt. Estimating the absolute position of a mobile robot using position probability grids. In National Conference on Artificial Intelligence, volume 2, pages 896-901, 1996.

[19] S. Thrun. A probabilistic online mapping algorithm for teams of mobile robots. International Journal of Robotics Research, 20:335-363, 2001.

[20] R. Kurazume, S. Nagata, and S. Hirose. Cooperative positioning with multiple robots. In IEEE International Conference on Robotics and Automation, volume 2, pages 1250$1257,1994$.

[21] E. W. Nettleton, P. W. Gibbens, and H. F. Durrant-Whyte. Closed form solutions to the multiple platform simultaneous localization and map building (SLAM) problem. In Proceedings of the SPIE, volume 4051, pages 428-437, 2000.

[22] K. Whitehouse and D. Culler. Macro-calibration in sensor/actuator networks. Mobile Networks and Applications, 8:463-472, 2003.

[23] N. Patwari, J. N. Ash, S. Kyperountas, A. O. Hero III, R. L. Moses, and N. S. Correal. Locating the nodes: cooperative localization in wireless sensor networks. IEEE Signal Processing Magazine, 22(4):54-69, July 2005.

[24] Haowen Chan, Mark Luk, and Adrian Perrig. Using clustering information for sensor network localization. In Viktor K. Prasanna, Sitharama Iyengar, Paul Spirakis, and 
Matt Welsh, editors, Distributed Computing in Sensor Systems, volume 3560, pages 109-125. Springer, 2005.

[25] Weiwei Sun. Lower bounds of the minimal eigenvalue of a hermitian positive-definite matrix. IEEE Transactions on Information Theory, 46(7):2760-2762, 2000.

[26] Daeyoung Park and Byeong Gi Le. On determining upper bounds of maximal eigenvalue of hermitian positive-definite matrix. IEEE Signal Processing Letters, 10(9):267-269, 2003.

[27] L.M. Delves. On the temple lower bound for eigenvalues. Journal of Physics A: General Physics, 5:1123-1130, 1972.

[28] A. I. Mourikis and S. I. Roumeliotis. Predicting the performance of cooperative simultaneous localization and mapping (c-slam). International Journal of Robotics Research, 25(12):1273-1286, December 2006.

[29] C. Godsil and G. Royle. Algebraic Graph Theory. Springer, 2001.

[30] B. Mohar. Laplacian spectrum of graphs. Graph Theory, Combinatorics and Applications, 2:871-898, 1991.

[31] Thomas J. Otahal and Herbert G. Tanner. An extended Kalman filter implementation for the Khepera II mobile robot. Technical Report ME-TR-08-001, The University of New Mexico, December 2008.

[32] E. Ivanjko, I. Petrović, and M. Vasšak. Sonar-based pose tracking of indoor mobile robots. Automatika, 45(3-4):145-154, 2004. 